\title{
Reusable and Recyclable Graphene Masks with Outstanding Superhydrophobic and
}

\section{Photothermal Performances}

Hong Zhong ${ }^{1}$, Zhaoran Zhu ${ }^{1}$, Jing Lin ${ }^{1}$, Chi Fai Cheung ${ }^{1}$, Vivien L. Lu ${ }^{2}$, Feng Yan ${ }^{3}$, Ching-Yuen Chan ${ }^{1}$, Guijun $\mathrm{Li}^{1 *}$

${ }^{1}$ State Key Laboratory of Ultra-Precision Machining Technology, Department of Industrial and Systems Engineering, The Hong Kong Polytechnic University, Kowloon, Hong Kong, China

${ }^{2}$ Department of Building Services Engineering, The Hong Kong Polytechnic University, Kowloon, Hong Kong, China

${ }^{3}$ Department of Applied Physics, The Hong Kong Polytechnic University, Kowloon, Hong Kong, China

Email: mitch.li@polyu.edu.hk

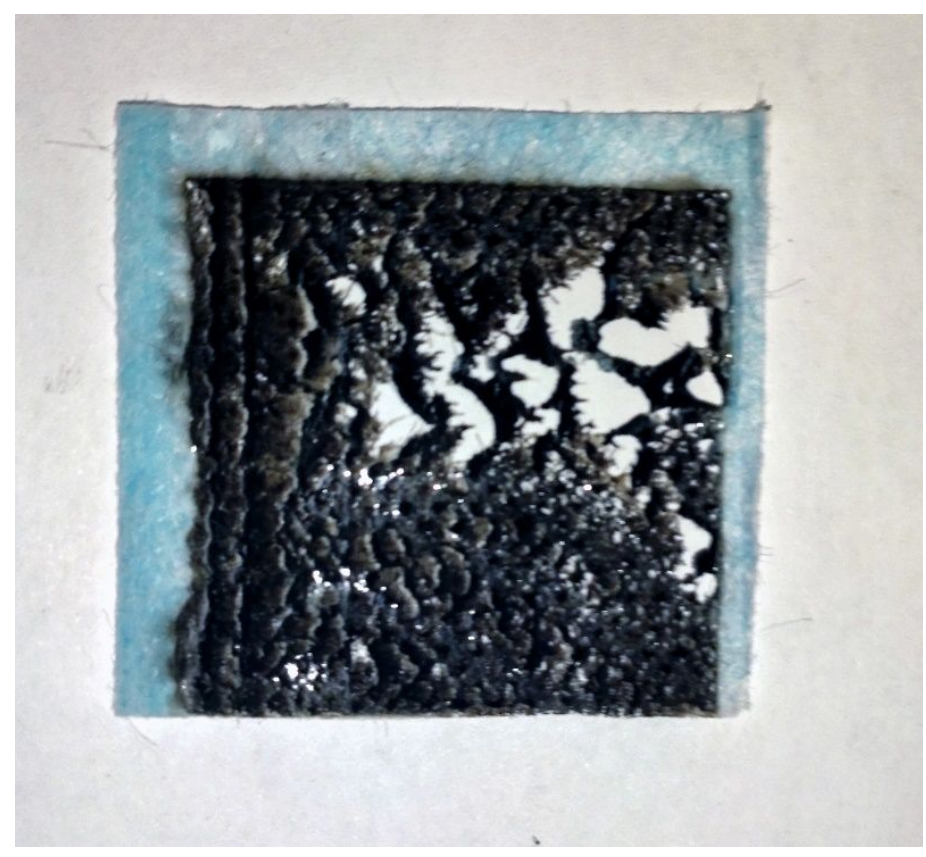

Figure S1. Optical image of the mask after $3^{\text {rd }}$ generation CW-LIFT graphene. Due to the high temperature with the $\mathrm{CW}$ laser, the mask was melted. 


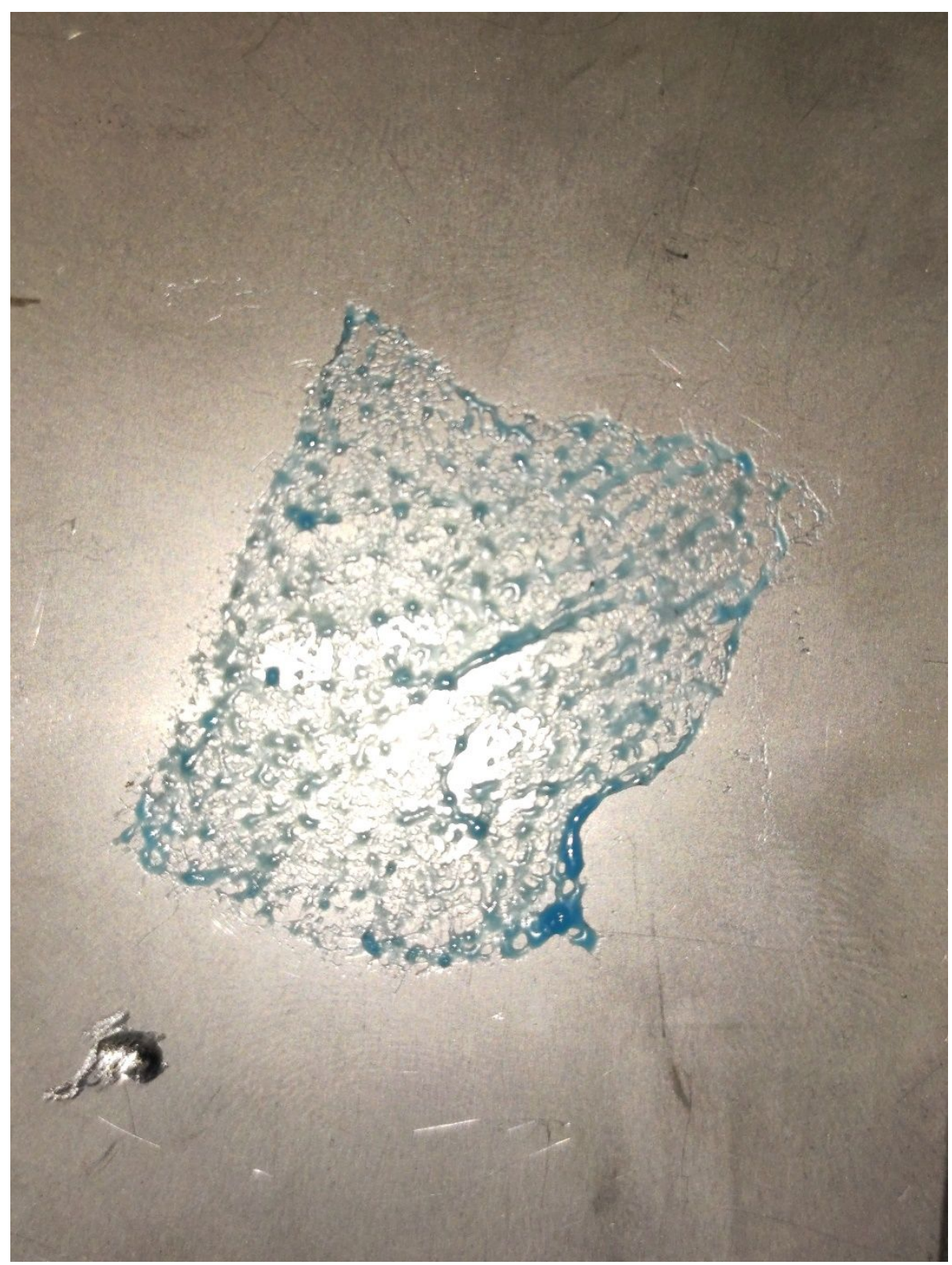

Figure S2. The optical image of the melted mask. After heating at $130^{\circ} \mathrm{C}$ using hotplate, the pristine mask had melted. 


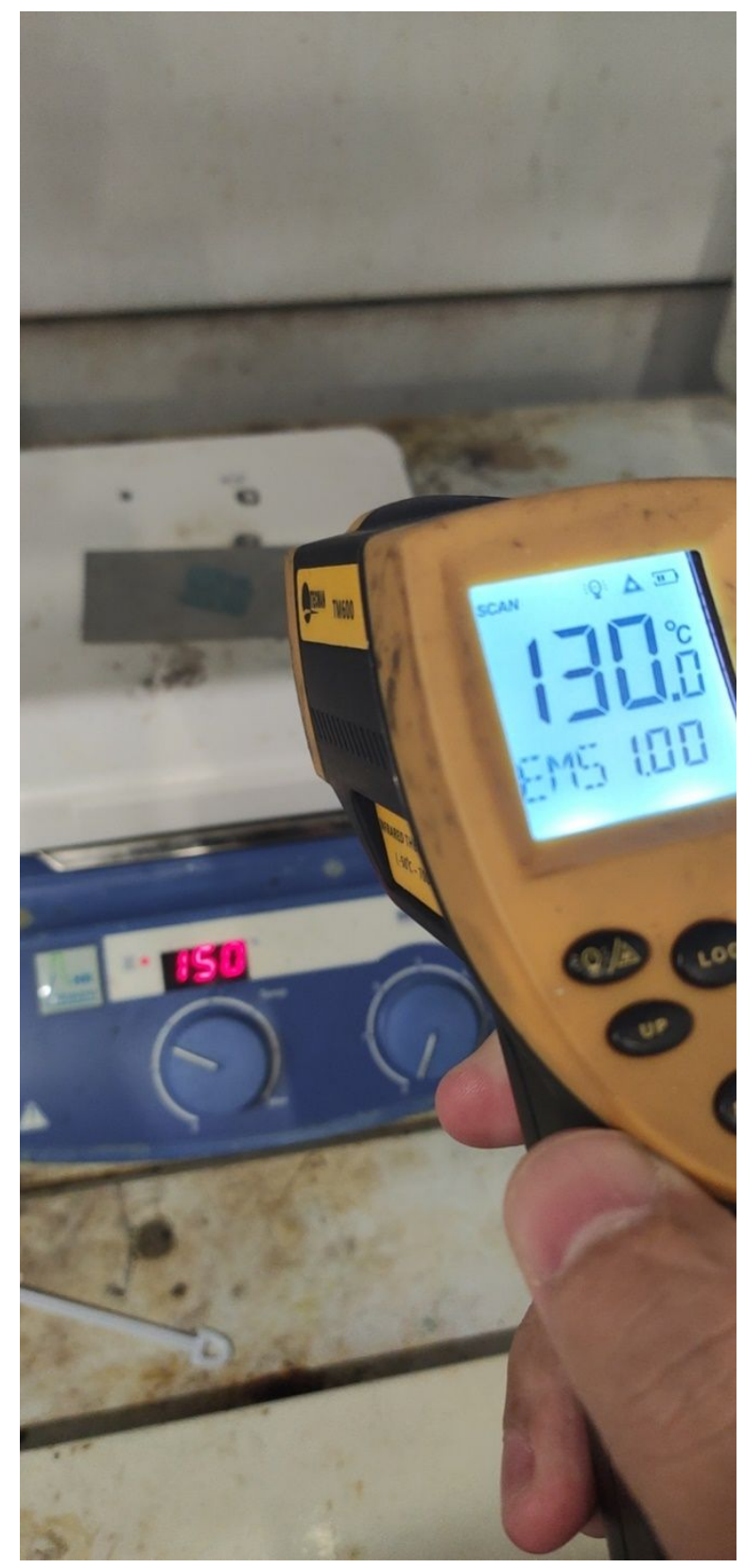

Figure S3. The experimental setup for measuring the melting point of the pristine mask. The hotplate was set to $150^{\circ} \mathrm{C}$, and the surface temperature of the pristine mask was measured to be $130{ }^{\circ} \mathrm{C}$. 

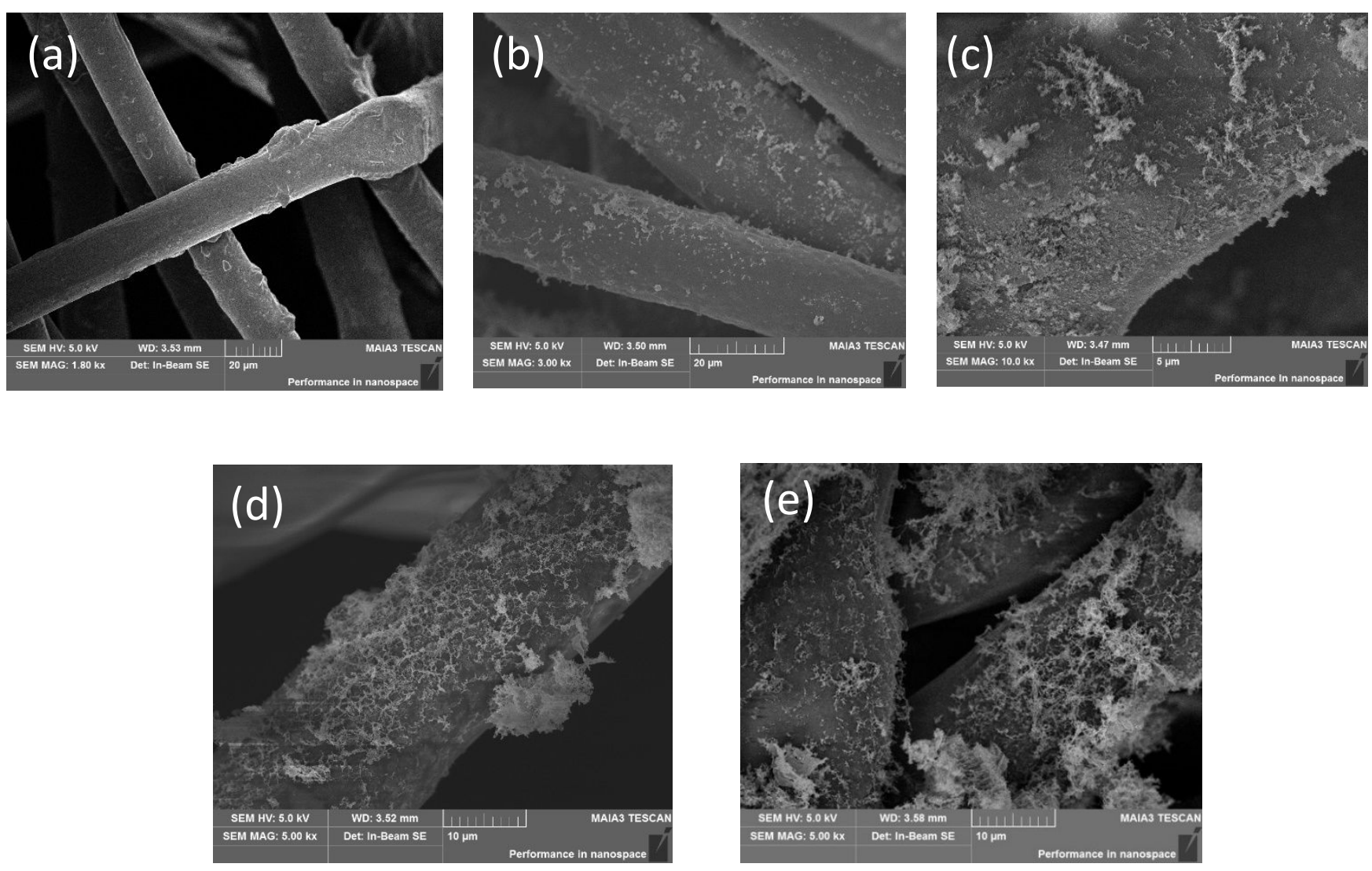

Figure S4. SEM images for (a) Pristine mask. (b) Mask with graphene no aging test. (c) Mask with graphene $24 \mathrm{~h}$ aging test. (d) Mask with graphene $48 \mathrm{~h}$ aging test. (e) Mask with graphene $72 \mathrm{~h}$ aging test. 

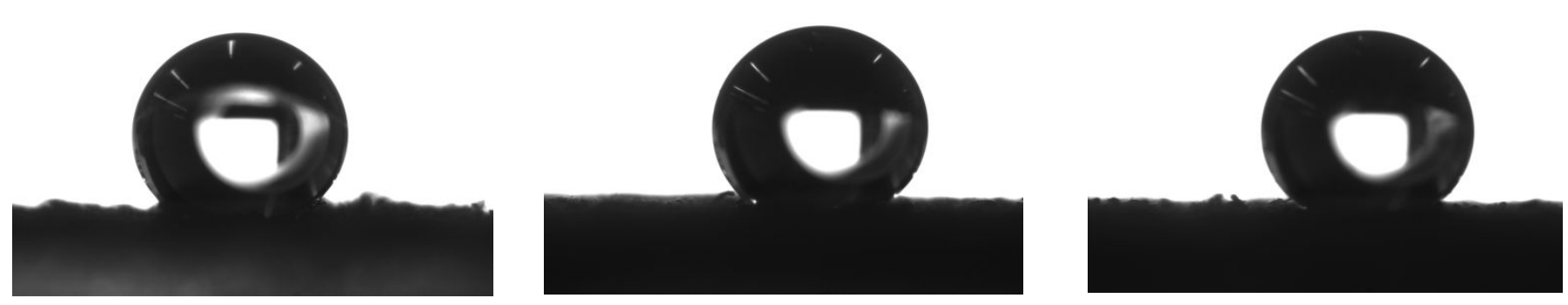

Figure S5. The water contact angles of the masks after solar sterilization after $24 \mathrm{~h}, 48 \mathrm{~h}$ and $72 \mathrm{~h}$ with 1 sun intensity. 


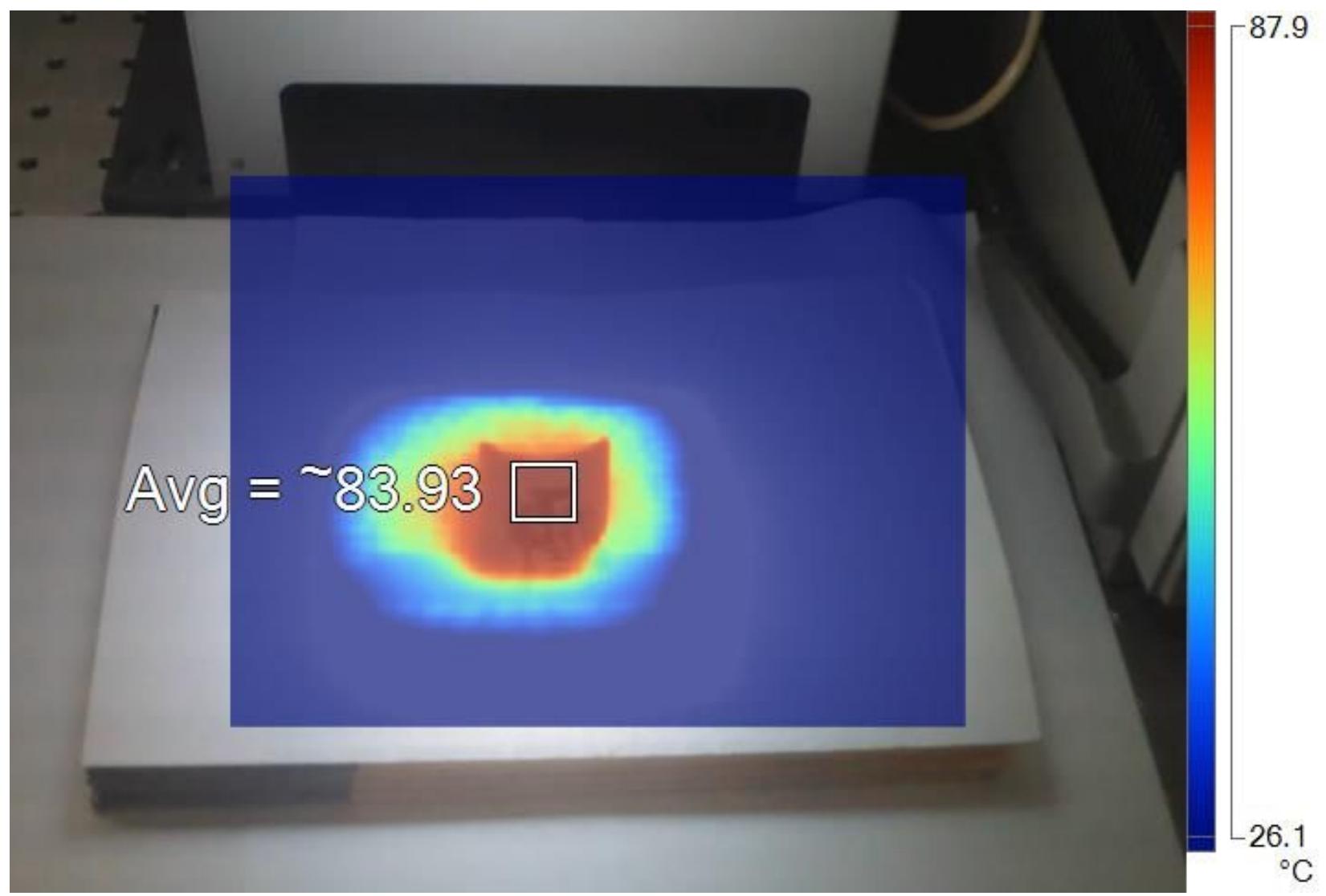

Figure S6. IR image of a $3.7 \mathrm{~cm}$ by $3.7 \mathrm{~cm}$ graphene coated mask sample after $72 \mathrm{~h}$ solar sterilization, under 1 sun intensity. 


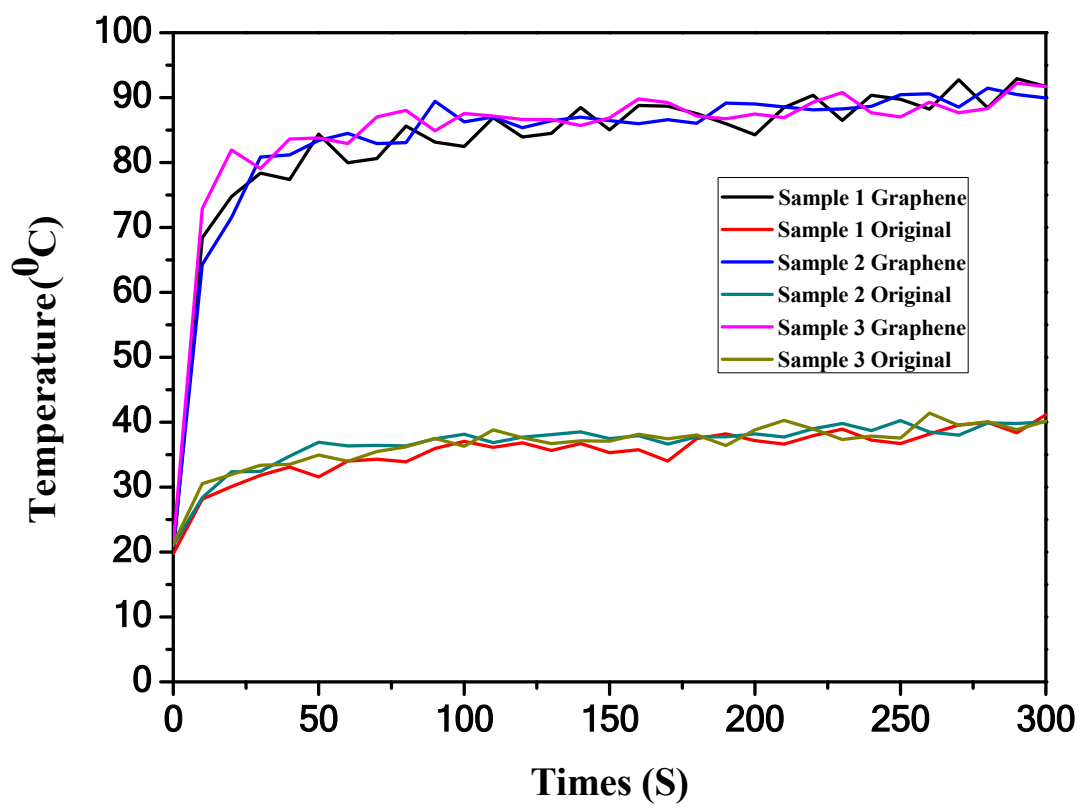

Figure S7. The measured temperature of graphene coated mask and the pristine masks against time. 


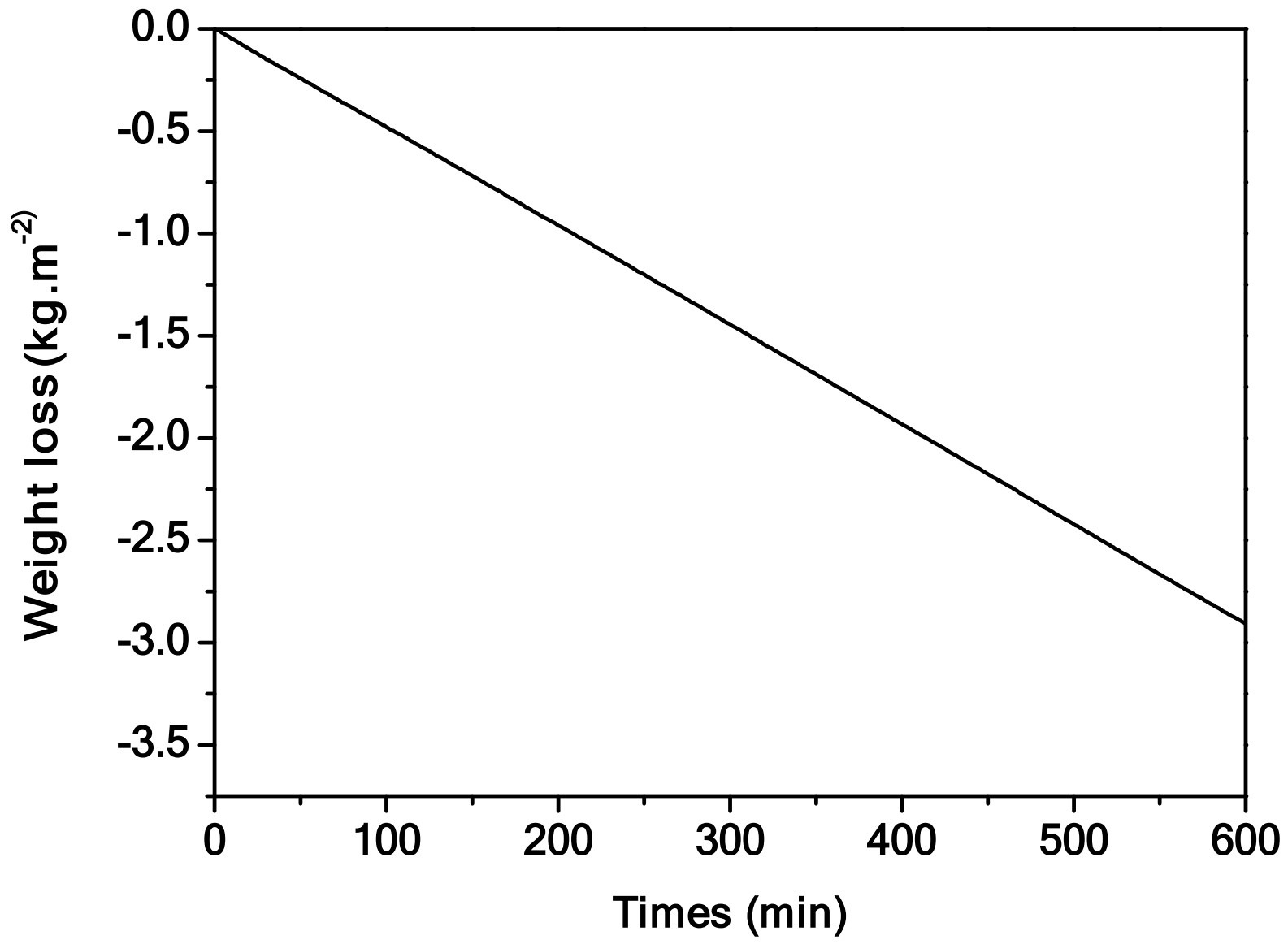

Figure S8. The desalination efficient of original mask, which is $0.29 \mathrm{~kg} / \mathrm{m}^{2} \mathrm{~h}$. 

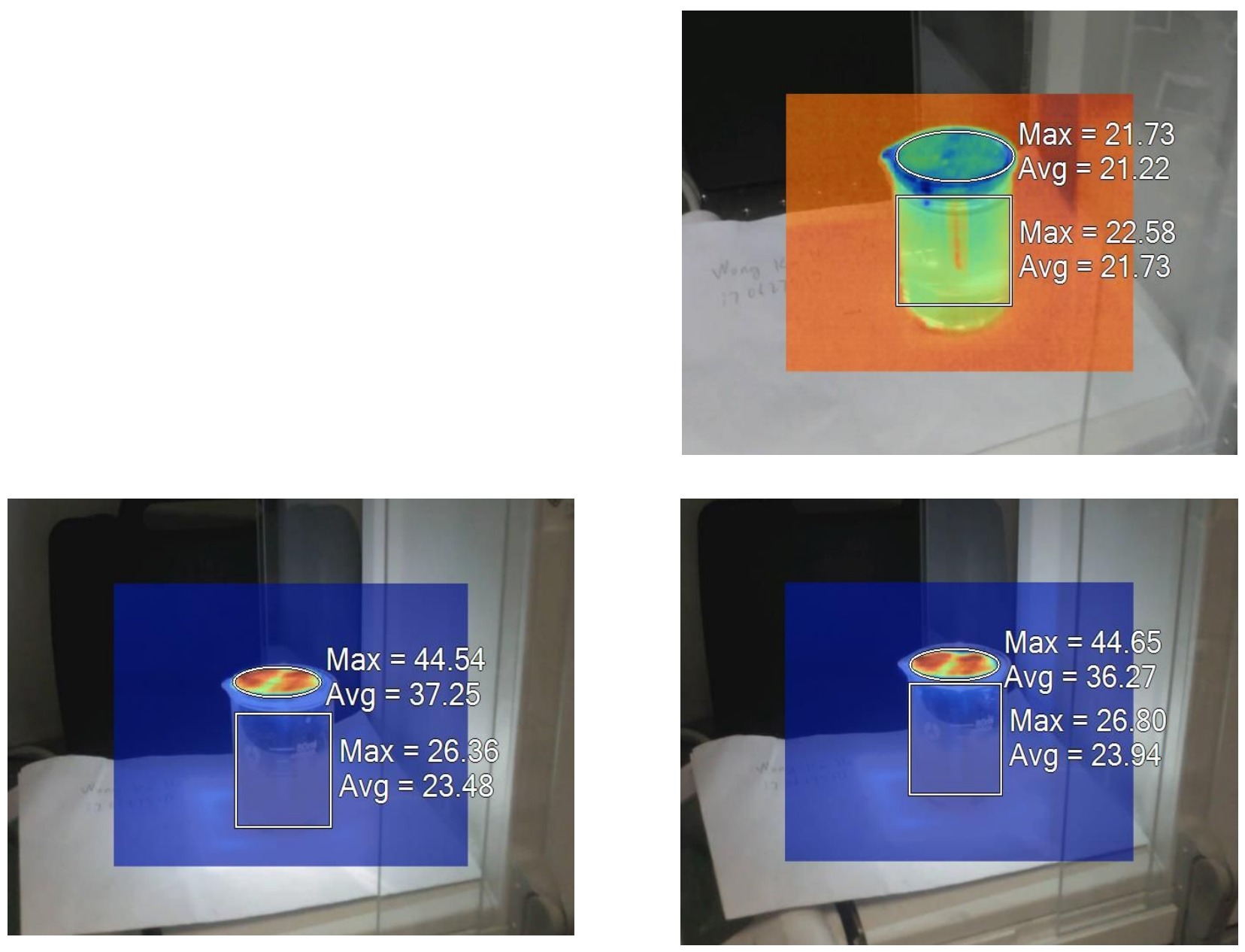

Figure S9. Solar steam generator (upper: before desalination; bottom: during desalination). 